\title{
VLBI OBSERVATIONS OF LOW-REDSHIFT RADIO GALAXIES
}

\author{
S.J. TINGAY ${ }^{1}$, D.L. JAUNCEY ${ }^{2}$, R.A. PRESTON ${ }^{3}$, D.L. MEIER ${ }^{3}$, \\ J.E. REYNOLDS ${ }^{2}$, A.K. TZIOUMIS ${ }^{2}$, J. LOVELL ${ }^{4}$, D.L. JONES ${ }^{3}$, \\ P.M. MCCULLOCH ${ }^{4}$, D.W. MURPHY ${ }^{3}$ AND G.D. NICOLSON ${ }^{5}$ \\ ${ }^{1}$ Mount Stromlo Observatory, Canberra, ACT 2611, Australia \\ 2 ATNF, Epping, NSW 2121, Australia \\ 3 Jet Propulsion Laboratory, Pasadena, CA 91109, USA \\ 4 University of Tasmania, Hobart, Tasmania 7001, Australia \\ ${ }^{5}$ HRAO, Krugersdorp 1740, South Africa
}

\section{Low-redshift, compact radio sources}

Here we will describe briefly some of the VLBI observations we are making of low-redshift, compact radio sources in the southern hemisphere, using the Southern Hemisphere VLBI Experiment (SHEVE) array of telescopes (Jauncey et al., 1994).

\section{PKS 0521-365}

PKS 0521-365 $(z=0.055)$ is a powerful radio source, with a strong radio core, extended structure in the form of a radio jet and hot spots (Keel, 1986). Coincident with the radio jet is an optical jet which has been observed with HST (Macchetto et al., 1991). PKS 0521-365 is the second lowest redshift EGRET identified radio source. Our VLBI observations (e.g. Figure 1) show a strong, unresolved core component and a pc-scale jet which aligns well with the kpc-scale jet. For more details see Tingay et al., ApJ submitted.

\section{PKS 1718-649}

PKS 1718-649 ( $z=0.014)$ may be the lowest redshift GPS radio source. Recent radio spectra show a turnover between 2.3 and $4.8 \mathrm{GHz}$. We have observed low flux density variability and low radio polarisation. Our VLBI 

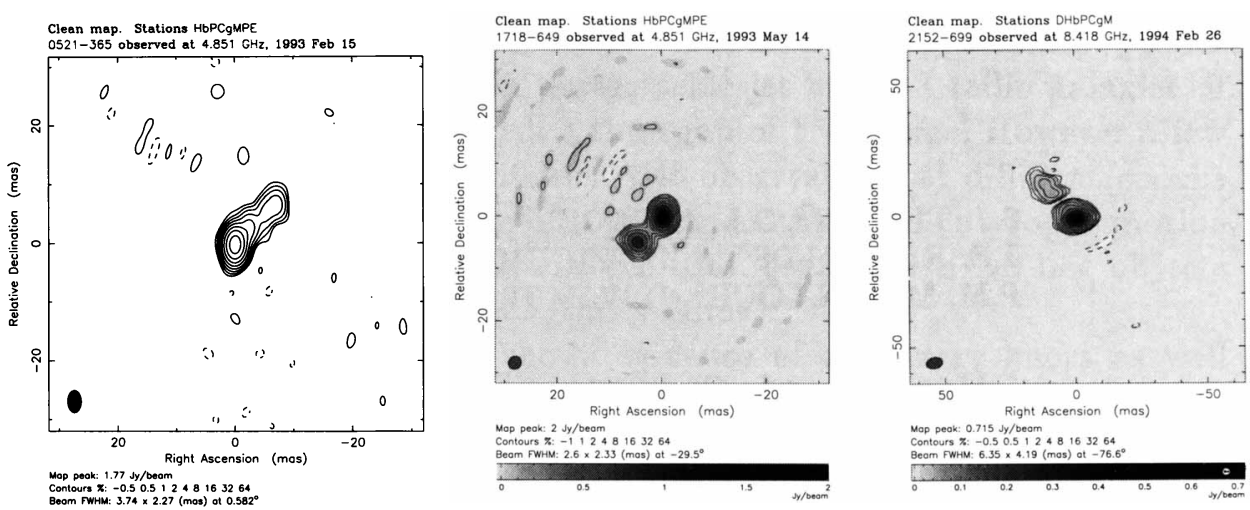

Figure 1. (left) SHEVE VLBI image of PKS 0521-265. (centre) SHEVE VLBI image of PKS 1718-649. (right) SHEVE VLBI image of PKS 2152-699

observations (e.g. Figure 1) show that the total flux density of the source is due to two compact components each approximately $0.5 \mathrm{pc}$ in extent (Tingay et al., in prep). From the HI observations of Véron Cetty et al. (1995) and the optical observations of Filippenko (1985) evidence can be found for confinement of the radio source by a dense, merger-induced environment.

\section{PKS 2152-699}

PKS 2152-699 $(z=0.028)$ is a powerful FR-II type radio source with an extra-nuclear emission line region which strongly resembles a typical nuclear emission line region (Tadhunter et al., 1988). Our VLBI observations (Figure 1) show that the pc-scale radio jet in this source is exactly aligned with the extra-nuclear emission line region. The jet is likely to be interacting with the galaxy in this region. For a more detailed discussion see Tingay et al., 1996, AJ, in press.

\section{References}

Filippenko, A.V., 1985, ApJ, 289, 475

Jauncey, D.L. et al., 1994, in Very High Angular Resolution Imaging (Eds J.G. Robertson and W.J. Tango), p 131

Keel, W.C., 1986, ApJ, 302, 296

Macchetto, F. et al., 1991, ApJ, 369, L55

Tadhunter, C.N. et al., 1988, MNRAS, 235, 403

Véron-Cetty, M.P. et al., 1995, $A \& A, 277$, L79 